\title{
Arbor
}

\section{El Viaje del Mundo de Pedro Ordóñez de Cevallos}

\section{Fernando Escribano Martín}

Arbor CLXXX, 711-712 (Marzo-Abril 2005), 581-594 pp.

Pedro Ordóñez de Cevallos, jienense que vivió entre mediados del siglo XVI y hasta la tercera década del XVII aproximadamente, pasó treinta y cinco años de su vida viajando, por todo el mundo si seguimos el título de uno de sus libros. De su periplo destacaremos sobre todo la parte a América y el Oriente Próximo.

Una vez regresado a su ciudad natal plasmó sus viajes en un libro (aunque escribió otros) y es este periplo del que hablamos en este trabajo. Existen, es cierto, dudas sobre el personaje y sobre sus fuentes, pero la magnitud de su testimonio, y los datos de primera parte que aporta, hacen de él un gran viajero a destacar.

Pedro Ordóñez de Cevallos, el «clérigo agradecido», tal y como él se hacía llamar en sus libros, viajó por el mundo a finales del siglo XVI y principios del XVII con distintas ocupaciones y objetivos. Fue soldado y corsario antes que sacerdote, incluso quizá después. Él se adjudica la catolización de miles de almas, e incluso cuenta cómo rechazó un reino por no renunciar a su condición eclesiástica. Su vida es sin duda propia de una novela de aventuras (de hecho, a veces, su libro parece más esto que una biografía) y quizá es en parte lo que pretendió trasmitir.

Uno de los principales problemas para trazar su biografía es que prácticamente la única fuente que manejamos es lo que él cuenta de sí mismo en su libro, que es también lo que han utilizado los que han tratado la figura y la obra del personaje. 
Al regreso de sus viajes, en Jaén, la ciudad que le vio nacer (hacia $1545^{1}$ ), escribió varios libros. Su Viage del Mundo, donde cuenta todos los viajes y aventuras que corrió a lo largo de su vida, publicado por primera vez en Madrid en 1614, y dividido a su vez en tres libros. Quarenta Triunfos de la Santísima Cruz de Christo N.S. y Maestro, publicado también en Madrid en 1614, que él tenía pensado que fuese el cuarto libro del anterior, pero le pareció que trataba cosas lo suficientemente distintas como para apartarlo. También realizó una historia de Jaén, que él, por enfermedad, no pudo terminar, y que concluyó y firmó Bartolomé Ximenes Patón, con el título de Historia de la Antigua, y continuada nobleza de la ciudad de Iaen, muy famosa, muy noble, y muy leal: guarda, y defendimiento de los Reynos de España. Y de algunos varones famosos, hijos della, publicado en Jaén en 1628. Publicó un Tratado de las Relaciones verdaderos de los Reynos de la China, Cochinchina y Champaa..., también en Jaén en 1628, y Tres entremeses famosos a modo de comedia de entretenimiento (Entremés del rufián; Entremés del astrólogo médico; Entremés del emperador y damas) publicados en Baeza en $1634^{2}$.

Por la historia de Jaén sabemos que cuando se publica el libro está convaleciente de una larga enfermedad, y que por eso delega su finalización. Algunas fuentes, de hecho, señalan que moriría hacia 1630. En este libro hay un capítulo, el 37 , dedicado a su figura, como uno de los hijos más insignes de la ciudad (¿sería idea suya incluirlo así o de Bartolomé Ximenes?) que se titula: Del Famoso soldado y Sacerdote Pedro Ordóñez de Seballos, primer autor desta historia, y en donde encontramos algún dato más de la vida del autor, pero en su mayoría tomados y celebrados de su Viage al Mundo.

La repercusión de su obra y el grado de conocimiento de la misma son relativas. Quizá, como señala Felix Muradas ${ }^{3}$, el hecho de que no fuese un descubridor ha menguado el peso de su fama, y quizá el que su libro contuviese pasajes sospechosos de ser inventados, o basados en otros, en una curiosa mezcla que desconcierta, que impide saber si el autor se deja llevar por su imaginación, o si sólo describe lo que vio, si es pura inventiva, o si narración real, también impidiese su mayor conocimiento y divulgación.

Es objeto de controversia la veracidad de lo que cuenta Ordóñez, incluso si visitó todos los lugares que describe en su libro. El mismo Muradas señala la sorprendente similitud de la tercera parte del libro con el «Itinerario» del Padre Martín Ignacio de Loyola, incluido como libro tercero en la Historia del Gran reino de la China de fray Juan González de Mendoza (Roma, 1585), y quizá haya más parecidos. 


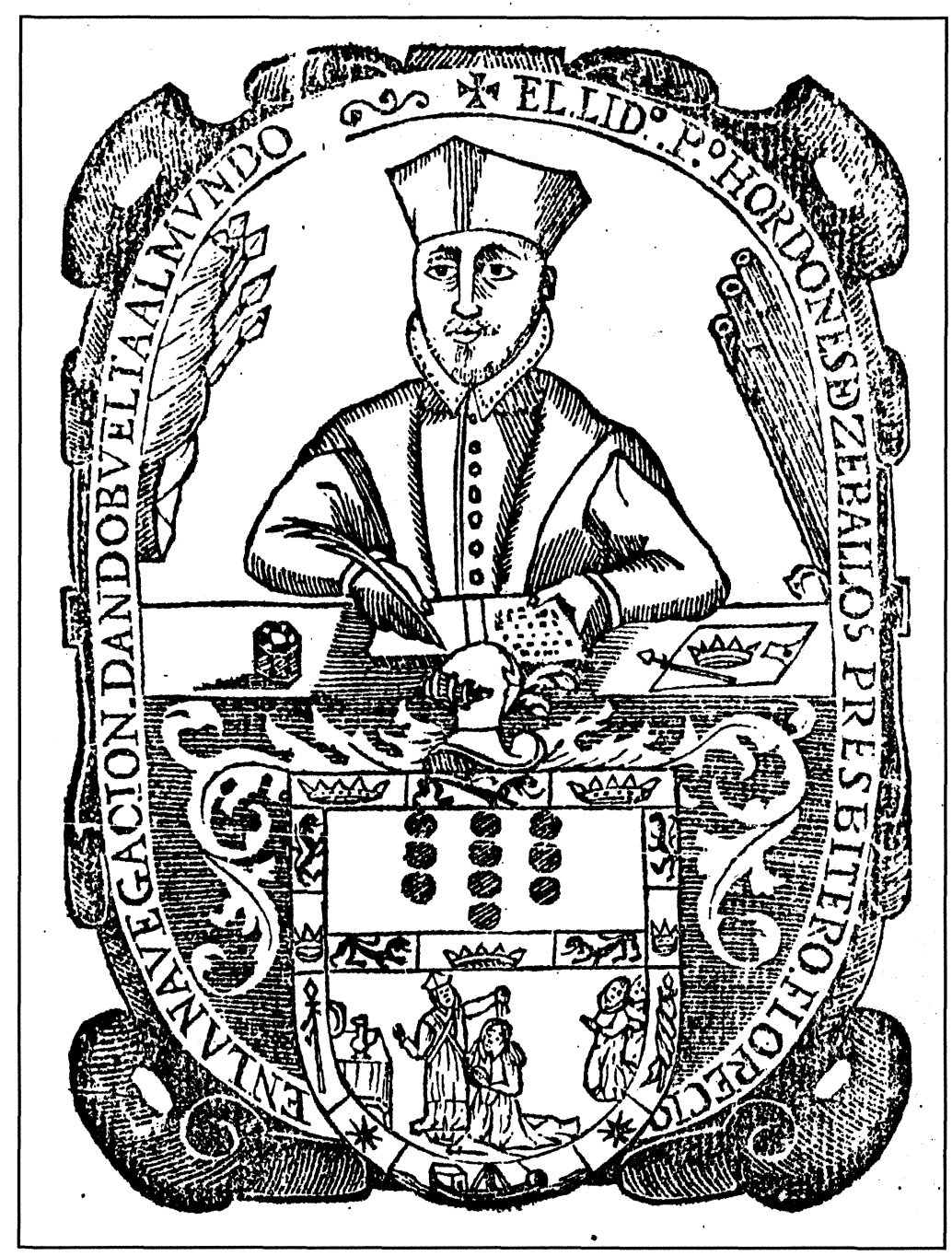

Frontispicio del libro de Ordóñez de Cevallos "Viaje del Mundo» 
Lo cierto es que sí se observa una importante repercusión de la obra en el siglo XVII, siendo reeditado en 1616 y 1691. Es también incluido en parte dentro de la Descripción de las Indias Occidentales de Antonio de Herrera (Amsterdan, en holandés en 1621; y en latín y francés en 1622), introducido así: Particuliere Description de L'Inde Occidentale, Touchant la fituation de les Terres \& Provinces, le chemin qu'il faut tenir à les paffer, \& quelles richeßes d'or \& argent fe trouven en chafcune d'icelles; Par le Prefte PEDRO ORDÓNEEZ DE CEVALLOS / Qui les recherche fort curieufement.

Posteriormente la obra apenas es citada o utilizada, pese a que contiene sin duda valiosísimos datos acerca de la sociedad, la geografía, los tipos humanos, la agricultura, la religión... de los territorios que recorrió. Quizá el evidente componente biográfico, pero también lo imaginativo a veces del relato le restó credibilidad, y pasó a un prudente, e injusto, olvido. Creo, sin embargo, que aunque ciertas partes del texto presenten alguna duda sobre su verosimilitud, que en cualquier caso no vamos a poder nunca comprobar, ofrece en su conjunto una estupenda radiografía del mundo (más de lugares como el reino de Nueva Granada, o de Indochina) a finales del siglo XVI, aporta muchísima información de primera mano sobre etnología, botánica, faunia, geografía, sociedades, cultos..., sobre cómo se descubría y extendía el poder español por América, cómo se organizaba el comercio y la explotación de los territorios y, en cualquier caso, es un fabuloso relato de viajes y aventuras, no sé si todas reales, pero sin duda todas apasionantes.

Vamos a tratar aquí, por lo tanto, con mayor amplitud, su libro Viage del Mundo, en el que describe la aventura que, durante treinta y cinco años le llevó a recorrer el mundo, y que escribió a su regreso en Jaén. Este libro está ordenado a la vez en tres. En el primero cuenta sus aventuras hasta que es ordenado sacerdote, ordenación que se celebró, según el final del primer capítulo del segundo libro, desde el día de Santa Lucía hasta el día de la dominica, in Pasione de todas órdenes. En el segundo trata lo que le sucedió desde que es ordenado sacerdote por el arzobispo de Santa Fe hasta que regresa a España, el final de la aventura que describe. El libro tercero, por último, contiene el itinerario y camino de todo el mundo y navegación de todo él, ordenado de un modo curioso: que primeramente pondré el camino derecho por donde se ha de andar y después por donde lo anduve $y o^{4}$.

Los grandes trazos de su viaje, jalonado de pasajes de su vida, muy resumido y escueto, se plasma en el capítulo biográfico de la historia de Jaén que hemos ya comentado, donde se describe el viaje a modo de pre- 
sentación, con la información obtenida, como dice el propio texto, del libro del autor. Incluimos aquí el párrafo, pues creo que explica de forma perfecta sus viajes:

Sumando 5 pues lo que en sus libros dixo, el nacio en Jaen de padres Chriftanos, y principales, donde nueve años, hafta diez y nueve, ò veynte fe crio en Sevilla, cafa de un tio fuyo donde eftudio Latinidad, y Artes en que fe grafuò. Por las cauffas que alli dize fe embarco al amparo de don Iuan de Cardona Genera de las Galeras de Efpaña en aquella razon, el qual dende fus principios le favorecio $y$, honro, haziendole primero alguacil Real de las galeras, y luego fu Alférez y defpues Capitan. En Cartagena de las Indias falio contra Negros Cimarrones, y los vencio, prendio, y apaciguò aquella tierra, y caminos. Fue Capitan contra navios de la Rochela y echo uno a fondo. En la jornada de Braba, y Caribana, fu Maeffe de Campo, y llevó treynta y feys hombres y feys Negros fuyos y focorriò el Real con comida y municion es en que gaftò mas del feys mil ducados. Vifitò las guarniciones de Antioquia, y Papayã: donde fue Gobernador, y en el interin apafiguò a aquella tierra de los Indios Pijaos, que la teniã oprimida, y focorriò otro Capitan en los Sutagaos, que lo tenian cercado, y fe poblo la Ciudad de Altagracia. Eftas hazañas por mayor, y otras innumerables por menor hizo en más de treinta años, que fue Seglar, defpues de falido de Sevilla. Auiendofe ordenado de Sacerdote fue Cura, y Vicario de la Ciudad de Pamplona, y Vifitador general en el nuevo Reyno de Granada. Despues hizo un viage dede la nueva Efpaña a la China, y junto a la Ifla de Ladrones defcubrio una Ifla de Efpañoles perdidos, donde hizo gran fervicio a Dios. En los Reynos de Cochinchina done fue prefo, enfeño y baptizo una hermana del Rey, que era Reyna de Campa, y otros Virreyes, Capitanes, y otras gentes, $y$ por ello eftuvo condenado a muerte, y lo defteraron. Retratò navios de chriftianos y les dio libertad, aunque le fue mal agradecido. De buelta peleò con turcos, y con Inglefes, de donde falio herido. Por buenos ayres boluio al Piru, y en la Provincia de Quito, por mandato de la Real Audiencia entro en la de los Quijos, que eftavan revelados, y pagò la gente a fu costa, y los apaciguò; y de los de guerra, Omaguas y otras naciones facò mas $d$ equince mil, y los viftio, por fer gente defnuda, cautiuan unos a otros refcatò muchs y les dio libertad, enfeño, baptizo y poblo: Y por ser tierra de guerra, montuofa, y de a pie con lodos, y aguaceros pafto grandes trabajos, y faco dos enfermedades que le duran hafta oy. Defpues, fue Cura, y Vicario dela Provincia de Pimampiro, donde enfeño, y baptizo mucha gente, y dio a las Iglesias cantiofas limofnas. (...)

De un recorrido así, que trascurre a lo largo de toda una vida, narrado y explicado con multitud de descripciones, aventuras, datos y anécdo- 
tas, surgen multitud de párrafos destacables en función de los temas que pretendamos resaltar, o que dejan a la luz visiones e impresiones del autor que sorprenden vivamente. En este texto, que no deja de ser una mera presentación, o recordatorio, del autor y de su obra, sólo vamos a entresacar algunos aspectos, dejando al lector el descubrimiento del libro en el libro mismo, como no puede ser de otra manera.

Uno de los asuntos que más espacio ocupa a lo largo del segundo libro, tanto en la descripción de su gestación, como en los comentarios al respecto, es la relación que el clérigo Ordóñez tuvo con la hermana del rey de Cochinchina, y reina ella así mismo de Champa ${ }^{6}$. A lo largo de varios capítulos, el autor y protagonista va describiendo cómo la relación entre él y la infanta se va afianzando, y cómo va creciendo el amor de ella hacia él. Él da la impresión de dejarse querer, y querer así mismo, pero su condición de sacerdote le impidió cualquier paso al respecto. Da, o quiere dar, la impresión de que el debate interno no es tanto el de sus sentimientos, a los que renuncia, sino a los beneficios que obtendría la Iglesia de tal relación, pues siendo él rey introduciría la fe católica en aquellos lejanos territorios. Llegó incluso a plantearse pedir bula papal ante la insistencia y amor verdadero de la infanta, solucionándose al final el tema con su religiosa negativa. Siendo luego ésta celebrada como un doble triunfo: a la carne, pues era la infanta joven doncella, y al poder y la gloria, pues lo que estaba en juego era un reino. Salió todo mejor de lo esperado, pues la infanta comprendió el porqué de su renuncia, y por seguirle, siquiera espiritualmente, y más acercarse a él, siquiera en condición, se hizo instruir en el catolicismo y después se bautizó, tomando para si la condición de monja. Varios miembros de su corte la siguieron en tal condición y fundó un convento, a modo de casa madre para la extensión de la fe en aquellas tierras.

Da también la impresión de que la infanta, convencida sin duda de su nueva espiritualidad, pretendía sobre todo que Ordóñez volviese, y así se trasluce en la correspondencia que mantuvieron y que se reproduce en el libro, en la que se mezclan noticias del país, del avance de la nueva fe, de la familia y de plegarias para su pronto regreso, en un tono que a veces no distingo si es espiritual o más carnal. Esta costumbre de incluir cartas que justifican y explican lo que narra, lo repite varias veces a lo largo del libro.

La infanta y reina, en su renuncia del mundo le entregó sus derechos a Ordóñez, lo cual se convertía sin duda en un problema político, que no llegó a más pues él se los entregó al hermano de la infanta y rey de Cochinchina, pudiendo decir desde entonces, como hace, que renunció a un reino. 


\section{El Viaje del Mundo de Pedro Ordóñez de Cevallos}

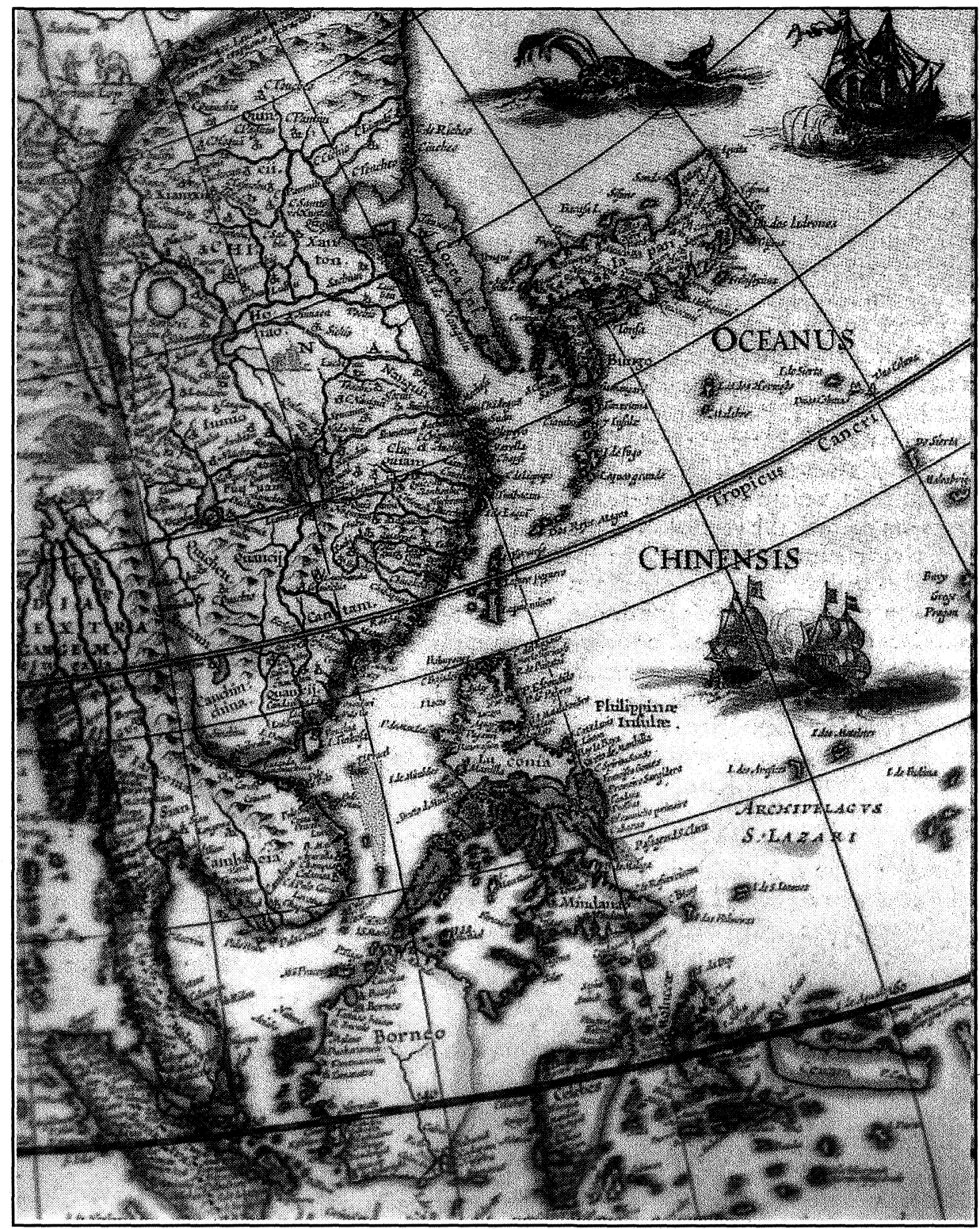

Mapa de Willem Blaeu (1617): Asia Noviter delineata

(detalle donde aparecen las tierras señaladas) 
Hecho éste de las conversiones que es sin duda central a lo largo del libro segundo, tanto en las Indias occidentales como en las orientales, pero que es difícil de comprobar, que si no daría la impresión que países enteros deben a él la buena nueva de la fe. Como muestra el título del capítulo XXXII: De la prosecución de estas naciones en hacerlas cristianas, hasta que envié todos los españoles.

En su recorrido, tal y como hemos visto, toca varias veces tierras del Oriente próximo. En el capítulo quinto del primer libro se cuentan los Lugares Santos que visitamos, en Jerusalén y alrededores, nombrando los lugares, con alguna pequeña descripción, como ilustración de la pasión de Cristo, sin entrar en mucho más detalle. En el capítulo siguiente, adonde se prosiguen las estaciones y lo demás que pasó en aquel santo viaje de Jerusalén, si entra más en el detalle de alguna de las principales iglesias, y de cómo se organizaban. Así, en la iglesia del Santo Calvario, que visitan:

"que es admirable edificio y el que más mueve a devoción y contemplación. Hay nueve géneros de frailes en esta iglesia, quiero decir de nueve naciones, y porque todos son de la religión del glorioso San Francisco; cada una va diferente en sus ceremonias y viven en paz, porque tienen pena de la vida si se entremèten los unos con los otros, y es lástima de ver tantos ritos en una sagrada fe, lo cual es parte para que los turcos sientan lo que les parece, de ver que cada uno diga que lo que él hace se ha de tener. ¡Dios los traiga a un verdadero conocimiento, pues son ovejas de un rebaño!

Esta visita a Tierra Santa es breve, y tras visitar Belén marcha a Túnez y Berbería. Regresa después a España y marcha a América. Para volver al Oriente próximo hemos de esperar hasta bien avanzado el libro segundo. El relato de Ordóñez de Cevallos permite conocer de los lugares que va recorriendo, y también de cómo se organizaba (cap. XXV):

Llegamos a la isla de Sumatra, que es enfrente de Malaca; es una isla grandísima y muy poblada. Hay en ella muchos señores; (...) Salimos de alli y vinimos a la isla de Micubar [Nicobar], que son dos islas pequeñas, y la una tiene un puerto, (...) De allí fuimos por un archipiélago de islas [debe tratarse de las islas Andamán -en nota-], que tenían a dos días y tres de camino, por aquel Gofo de Bengala, (...) Al fin de marzo llegamos a una isla pequeña que se llama Tanaceri, que es del rey de Siam, (...) En un golfo hay tres ciudades grandes y de mucha gente; llámase ésta Andilipatán, la otra Pipilipatán y la otra Pobilipatán; son del Gran Mogol y de grandísima contratación. (...)

Pasa después a Ceilán, donde es tomado prisionero y describe la isla?: Hay fama que en aquella tierra de Ceilán hay mucho género de riquezas, plata y oro, perlas, (...) Cógese infinito arroz, trigo, maíz y otras legumbres. Es la 


\section{El Viaje del Mundo de Pedro Ordóñez de Cevallos}

isla de quinientas islas de boj y muy fuerte; está partida en cinco señores; los cuatro no obedecen a nadie, y el uno, que cae en la mejor tierra y en la más, obedece al de los mogoles, y nuestro rey tiene alli cinco puertos...

Y de aquí a Goa (un 10 de julio), que es una hermosa ciudad, grande, rica, de más de veinte mil portugueses, y de la tierra más de cincuenta mil; tiene más de treinta y tantas iglesias, y entre ellas quince parroquias; ésta es una isla pequeña, pero es toda ella un jardín; hay lindas aguas y mantenimientos y muchos y baratos; es la cabeza de todo lo que allí está de Indias; el virrey de allí es más venerado que un gran rey; es ciudad riquísima y de gran contratación; está allí una casa y templo de la Compañia de Jesús, que es para ver; hayla también de los padres de Santo Domingo, San Francisco y San Agustín.

El recorrido que plantea el capítulo XXVIII es de vértigo: De cómo llegamos a Ormuz y de otras cosas diversas que nos sucedieron hasta llegar a Quito. Salen de Goa, (...) Llegamos ${ }^{8}$ a Diul, una fortaleza de Persia, de un sátrapa; diérannos salvoconducto para ls guardas, que nos dejaron pasar. Otro día que salimos de allí, que fue a veintitrés de agosto, llegamos a Ormuz, y estuvimos en ella hasta diez y nueve de septiembre... Después a Madagascar, y después por Arabia: Caminamos por aquella costa de Arabia sin engolfarnos otros cinco días, y a la vista de la isla de Cacatora (Socotora) descubrimos cinco galeras...

De Mataca, casi parecería que por encanto, llegaron, cuando se dirigían a España, a Brasil. Sucediónos el más prospero viaje que habiamos jamás llevado; tuvimos viento en popa y tan recio que sin saber ni poder tomar altura ni paraje caminando de día y de noche; al fin de mes nos hallamos en Hernanbuco [Pernambuco], un puerto de la isla de Brasil, de que nos quedamos espantados de la travesía que llevamos, y certificó el piloto que habiamos corrido más de mil y doscientas leguas, que me pesó grandemente, porque toda la gente junta acordó de no venir a España, sino de alli, por el estrecho de Magallanes, irse al Perú (...)

En el capítulo XII del libro tercero, dentro del Itinerario del viaje realizado que supone este libro, $A$ donde se tocan las cosas famosas del Gran Mogol y sus reinos señala que: Todos estos reinos, dentro y fuera del gran río Ganges, son del Gran Mogol. Nacen de una sierra los cuatro ríos famosos, $y$ otros me dijeron que nacían en una laguna y que eran siete, y están en la Tartaria, de que tiene este gran príncipe mucha parte ganado; $y$ se decía por muy cierto que el rey que lo era tan valeroso y guerrero que había ganado doce reinos y tres imperios, sin lo heredado de sus pasados desde el gran Taborlán [Tamerlán], Bayaceto, gran turco, y traerle en una jaula; cuando subía a caballo subía en la jaula, habiendo sido su primer estado 
un pobre pastor, que haciendo unos juegos lo eligieron por rey y de allí lo vino a ser de veras con gente que se le llegó; y desde la Trapisonda [Trebisonda] y otros sultanatos ganó y es suya la mayor parte de la Persia y Tartaria, y todos los más reinos de la India, hasta el de Perú (...)

Este párrafo muestra que Ordóñez de Cevallos conocía el viaje y el libro de González de Clavijo ${ }^{10}$ sobre su visita a la corte del Tamerlán a principios del siglo XVI. La leyenda de la jaula de Bayaceto con la que se apoyaba para subir al caballo, los cuatro ríos sagrados que nacen de un lugar común, y los reinos que llegó a conquistar es la misma información que se puede encontrar en Clavijo. Estos datos no creo que los recogiese in situ, sino que pertenencen a una labor, seguramente posterior, de documentación. Bien es cierto que su erudición, tal y como demuestra en sus libros, es altísima.

Ya hemos comentado que la parte más importante de sus viajes y estancias trascurren en América, aunque aquí hayamos desarrollado más algunos pasajes de su trascurso en el Oriente próximo. De hecho, es la parte que Antonio de Herrera incluye en el libro ya citado, publicado en Ámsterdam ocho años después del libro de Ordóñez de Ceballos, y que sería por lo tanto la parte que se conocería en Europa, y que muestran el crédito que se le dio en su momento a su obra.

Cuando en el libro tercero habla del viaje en el que regresaba a España y que le llevó y entretuvo en América, lo narra así1 ${ }^{11}$ : Y porque esta es la vuelta a todo el mundo y la que yo di fue vuelta y media, acabaré el viaje como lo anduve, que de Cabo de Buena Esperanza se engolfó mi galeón San Pedro y con temporal y hartos trabajos descubrimos las islas del Brasil y la gente no quiso volver a España (como narra igual en el libro segundo y hemos citado). Recorren, entre otros lugares: el Río de Plata, Paraguay, Tucamán, el Callao de Lima y Guayaquil. De aquí a Panamá, que su puerto es Perico. De esta ciudad se viene por tierra veinte leguas a Portobelo (...) y este Portobelo es en la mar del Norte; para venir a España hasta este puerto de la Habana, islas Terceras, Sanlúcar de Barrameda, Sevilla y Jaén, de donde partí de nueve años y gasté treinta y nueve en estas peregrinaciones, dando vuelta y media al mundo y habiéndole visto $y$ andado en mar y tierra sobre treinta y tres mil leguas. ¡Sea a honra y gloria de Nuestro Señor Jesucristo, que vive y reina por siempre jamás! Amén.

Así concluye en el capítulo XV el relato de sus viajes, dando pié a una serie de capítulos en los que da toda una lista de noticias curiosas y anecdóticas, pero también datos e informaciones interesantísimas que ha ido recopilando a lo largo de sus viajes. 


\section{El Viaje del Mundo de Pedro Ordóñez de Cevallos}

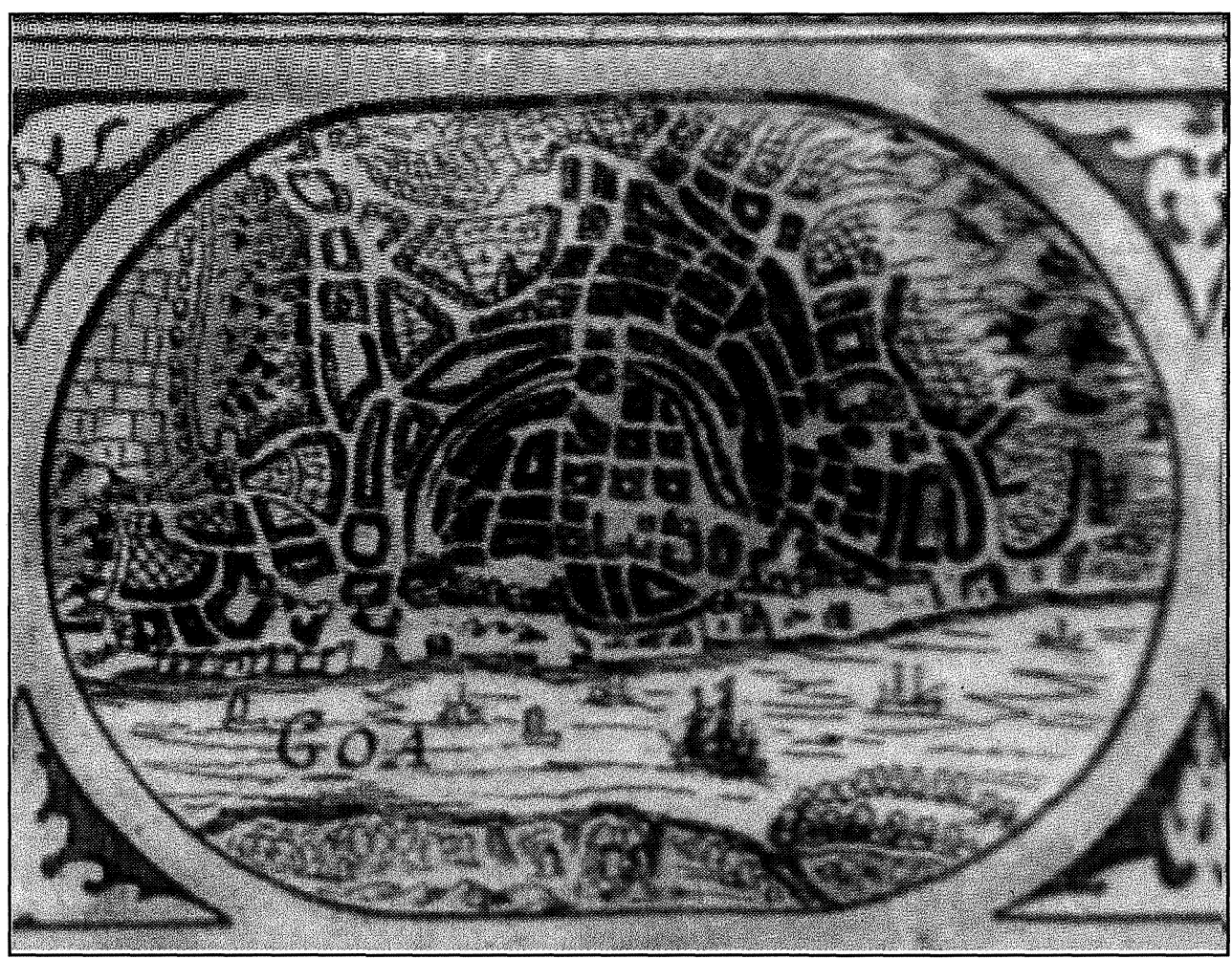

Plano de Goa que aparece en el mapa antes señalado 
Uno de estos capítulos, el XVI, Adonde se ponen las vidas y muertes de algunos varones santos que en aquellas partes de la India padecieron tormentos, en el que se refiere a las indias orientales, empezando a contar estas vidas y martirios por Francisco Javier, y tras él enumera a los padres y hermanos que allí murieron, pues es tanto lo que por allí padecen los que emprenden este ministerio, que no es explicable, si no se ve. Yo digo por testigo de vista ..., destacando, entre todas, como señala al final, la Compañía de Jesús.

Ya hemos comentado que el libro es una estupenda radiografía del mundo a finales del siglo XVI y principios del XVII. Fuesen las fuentes documentales de Ordóñez de Cevallos de un tipo a otro, se basase más o menos en otros autores, lo cierto es que a lo largo del libro, y especialmente en una especie de resumen en los capítulos XVII y XVIII del tercer libro (De la diversidad de reinos que hay en el mundo, de sus vasallos $y$ grandeza), al modo de lo que hizo Anthony Sherley (aproximadamente en 1622) con su Peso Político de Todo el Mundo ${ }^{12}$, glosa un pequeño comentario de las monarquías más importantes o por él conocidos. Tratando China, el Gran Mogol, el reino de Camboya, el Gran Catay, tártaros, el Gran Sofí, el Decán o Narsinga, Pegú, Siam y Camboya, Cochinchina, Japón, Sumatra: Después África: el reino de los abisinios, que llamamos del Preste Juan, Berberia. Eso sí: la otra parte mejor del mundo es Europa, de gente valerosísima y valiente y más sabia; contiene en sí al Gran Turco, que por ser notoria su grandeza pasaré por ella, aunque da algunos datos. Y trata Alemania, Italia, Francia, Inglaterra. Trata después lo que llama las Españas, refiriéndose a las coronas de lo que hoy es España, y la de Portugal, que es el mayor piélago que se ha visto.

Las Españas tienen casi nueve millones de vasallos, y por ser del católico Rey, Gran León de España, y ser un piélago sus señoríos y, como he dicho en la historia, es mayor señor del mundo, como se verá en lo que sigue...

De Portugal, y del poder del rey, da una imagen cierta de su extensión: La Corona de Portugal es el mayor piélago que se ha visto, pues tiene vasallos en todas las más partes del mundo, porque tiene en África, Terceras, Madeira, Brasil, Guinea, Mozambique, Ormuz, Persia, la India, Cambaya, Cochin, Pesquerías hasta cabo de Comori, Ceilán, Malipur, Malaca, Camboya, Macao y en infinidad de islas, que se dice que no hay reino ni provincia que toque en la mar que en más de cuatro mil leguas por esta parte y más de tres mil por la otra que en todos tenga el gran Rey e España tierra y puertos con pensiones para ellos, que se puede decir vasallaje, como en sus lugares he tocado. 
Después dará y hablará de cosas maravillosas del mundo, incluyendo toda una serie de animales sorprendentes para la época. Y terminará con cuatro capítulos referentes a América, donde tocará desde descripciones de los reinos, en un territorio que se está todavía descubriendo y se encuentra en plena expansión, temas sobre la organización social en aquellos territorios y la etnografía del mismo, sobre los obispados y sus gobiernos, y sobre la importancia de la cristiandad de la Nueva España. Terminando así el libro.

Esta obra de Ordóñez de Cevallos, como hemos ya insistido, nos presenta el mundo por el que se movió en el momento en el que lo vivió, suponiendo por lo tanto una fuente de primera mano que aporta un importante elenco de datos, de impresiones, de análisis y de descripciones únicas. En un momento en el que las coronas españolas se expanden por el mundo, por las Indias orientales gracias a la Corona de Portugal, y por América (las Indias occidentales), que está descubriéndose y colonizándose por iniciativa de la castellana. El autor las ha visitado y parece que ha participado de esta política de expansión en una posición preferente. Lo que pueda haber de fantasioso o tomado de otras fuentes, si es que es así, creo que se compensa por lo que tiene de apasionante el relato, y por la cantidad de información que aporta. Su vida fue, y es lo que escribió, una aventura. Su aventura fue su Viaje del Mundo.

\section{Bibliografía}

A lo largo del texto hemos señalado las obras de Ordóñez de Ceballos, y las ediciones que de su Viaje del Mundo se hicieron, así como lo que se incluyó en la obra de Herrera.

La edición moderna del libro Viaje del Mundo de Ordóñez de Ceballos es la que ha llevado a cabo Miraguano Ediciones y Ediciones Polifemo en Madrid en 1993, incluida en la Biblioteca de Viajeros Hispánicos.

No existe además de estos libros señalados muchos más trabajos sobre el personaje y su obra, por el olvido al que nos hemos referido. Sí se incluye un capítulo, tras una breve introducción, en Gerardo González de Vega: Por donde el viento nos lleve. Antología de relatos españoles de piratas. Barcelona 2002.

\section{Notas}

1 Así lo señala Félix Muradas en la introducción a la edición de Miraguano (Madrid 1993), los datos oscilarían entre 1530 ó 1531 y 1547.

2 Citados por Muradas en la introducción antes señalada.

3 Op.cit.

4 Prologo al tercer libro. 
5 Hemos mantenido en este texto la sintaxis y la ortografía de la época.

6 En el mapa Asia noviter delineata, que se remonta a 1617, de Willem Blaeu, junto a la Cochinchina aparece el territorio separado de Cambaoia, Malaca y Sian, agrupados por el mismo color. En el de India quae Orientalis dicitur et Insulae adyacentes, de 1635, el territorio de Tsiampa aparece separado del de Cambodia y Siam, al sur también de Cochinchina, aquí agrupado dentro de China, aunque aparece la ciudad en la frontera. Estamos hablando por lo tanto de un territorio que hoy ocupan en parte Vietnan y Laos.

7 Edición de Miraguano, p. 299.

8 Edición de Miraguano, p. 307. Entiendo que Diul es Dizjul, punto clave en este camino.

${ }^{9}$ Lo que señalamos entre corchetes es lo que así viene señalado en la edición de Miraguano, que es la que hemos utilizado para los textos, excepto cuando señalamos lo contrario.

10 Se pueden consultar las ediciones de la «Embajada a Tamerlán» de Ruy González de Clavijo de Francisco López Estrada, Clásicos Castalia, Madrid 1999; y la de Miraguano, Libros de los Malos Tiempos 8, con introducción de Ramón Alba, también en Madrid 1999.

11 Edición de Miraguano, p. 434.

12 Ver por ejemplo, Peso político de todo el mundo del Conde D. Antonio Xerley. Carmelo Viñas y Mey. C.S.I.C. Madrid, 1961. 